\title{
Toeplitz quantization on Fock space
}

\author{
Article
}

Accepted Version

Creative Commons: Attribution-Noncommercial-No Derivative Works 4.0

Bauer, W., Coburn, L. A. and Hagger, R. (2018) Toeplitz quantization on Fock space. Journal of Functional Analysis, 274 (12). pp. 3531-3551. ISSN 0022-1236 doi: https://doi.org/10.1016/j.jfa.2018.01.001 Available at https://centaur.reading.ac.uk/84024/

It is advisable to refer to the publisher's version if you intend to cite from the work. See Guidance on citing.

To link to this article DOI: http://dx.doi.org/10.1016/j.jfa.2018.01.001

Publisher: Elsevier

All outputs in CentAUR are protected by Intellectual Property Rights law, including copyright law. Copyright and IPR is retained by the creators or other copyright holders. Terms and conditions for use of this material are defined in the End User Agreement.

\section{www.reading.ac.uk/centaur}

\section{CentAUR}

Central Archive at the University of Reading

Reading's research outputs online 


\title{
TOEPLITZ QUANTIZATION ON FOCK SPACE
}

\author{
W. BAUER, L.A. COBURN, AND R. HAGGER
}

\begin{abstract}
For Toeplitz operators $T_{f}^{(t)}$ acting on the weighted Fock space $H_{t}^{2}$, we consider the semi-commutator $T_{f}^{(t)} T_{g}^{(t)}-T_{f g}^{(t)}$, where $t>0$ is a certain weight parameter that may be interpreted as Planck's constant $\hbar$ in Rieffel's deformation quantization. In particular, we are interested in the semi-classical limit

$$
\lim _{t \rightarrow 0}\left\|T_{f}^{(t)} T_{g}^{(t)}-T_{f g}^{(t)}\right\|_{t} .
$$

It is well-known that $\left\|T_{f}^{(t)} T_{g}^{(t)}-T_{f g}^{(t)}\right\|_{t}$ tends to 0 under certain smoothness assumptions imposed on $f$ and $g$. This result was recently extended to $f, g \in \mathrm{BUC}\left(\mathbb{C}^{n}\right)$ by Bauer and Coburn. We now further generalize $(*)$ to (not necessarily bounded) uniformly continuous functions and symbols in the algebra $\mathrm{VMO} \cap L^{\infty}$ of bounded functions having vanishing mean oscillation on $\mathbb{C}^{n}$. Our approach is based on the algebraic identity $T_{f}^{(t)} T_{g}^{(t)}-T_{f g}^{(t)}=$ $-\left(H_{\bar{f}}^{(t)}\right)^{*} H_{g}^{(t)}$, where $H_{g}^{(t)}$ denotes the Hankel operator corresponding to the symbol $g$, and norm estimates in terms of the (weighted) heat transform. As a consequence, only $f$ (or likewise only $g$ ) has to be contained in one of the above classes for $(*)$ to vanish. For $g$ we only have to impose $\lim \sup _{t \rightarrow 0}\left\|H_{g}^{(t)}\right\|_{t}<\infty$, e.g. $g \in L^{\infty}\left(\mathbb{C}^{n}\right)$. We prove that the set of all symbols $f \in L^{\infty}\left(\mathbb{C}^{n}\right)$ with the property that $\lim _{t \rightarrow 0}\left\|T_{f}^{(t)} T_{g}^{(t)}-T_{f g}^{(t)}\right\|_{t}=$ $\lim _{t \rightarrow 0}\left\|T_{g}^{(t)} T_{f}^{(t)}-T_{g f}^{(t)}\right\|_{t}=0$ for all $g \in L^{\infty}\left(\mathbb{C}^{n}\right)$ coincides with VMO $\cap L^{\infty}$. Additionally, we show that $\lim _{t \rightarrow 0}\left\|T_{f}^{(t)}\right\|_{t}=\|f\|_{\infty}$ holds for all $f \in L^{\infty}\left(\mathbb{C}^{n}\right)$. Finally, we present new examples, including bounded smooth functions, where $(*)$ does not vanish.
\end{abstract}

\section{INTRODUCTION}

For a suitable family of functions $\mathcal{F}$ and Poisson bracket $\{\cdot, \cdot\}$, one can consider the deformation quantization (in the sense of Rieffel [18, 19]) $f \mapsto T_{f}^{(t)}$, where $t \sim \hbar>0$ is a weight parameter and $\left\{T_{f}^{(t)}: f \in \mathcal{F}\right\}$ consists of linear operators on an appropriate Hilbert

Date: August 25, 2017.

2010 Mathematics Subject Classification. Primary: 47B35; Secondary: 30H20, 81S10.

Key words and phrases. Toeplitz operators, Fock space, semi-commutator, semi-classical limit, heat transform, oscillation.

The first and third author acknowledge support through DFG (Deutsche Forschungsgemeinschaft), BA 3793/4-1. 
space. Hereby essential are the following limit conditions:

$$
\begin{aligned}
\lim _{t \rightarrow 0}\left\|T_{f}^{(t)}\right\|_{t} & =\|f\|_{\infty}, \\
\lim _{t \rightarrow 0}\left\|T_{f}^{(t)} T_{g}^{(t)}-T_{f g}^{(t)}\right\|_{t} & =0, \\
\lim _{t \rightarrow 0}\left\|\frac{1}{i t}\left[T_{f}^{(t)}, T_{g}^{(t)}\right]-T_{\{f, g\}}^{(t)}\right\|_{t} & =0 .
\end{aligned}
$$

A typical approach to obtain such a quantization is to consider a family of weighted probability measures $\mu_{t}$ on some domain $\Omega \subseteq \mathbb{C}^{n}$ with corresponding Lebesgue space $L^{2}\left(\Omega, \mu_{t}\right)$ and then associate a Toeplitz operator $T_{f}^{(t)}$ to every $f \in \mathcal{F}$. This construction was considered for functions on several different domains $\Omega$ and results like (1.1) have been obtained under certain smoothness assumptions on the functions $f$ and $g$ (see [9, 10, 11, 12, 13, 14, 15, 16, 17]).

In this paper we are interested in the case where $\Omega=\mathbb{C}^{n}$ and $\left\{\mu_{t}\right\}_{t>0}$ is a family of Gaussian measures defined below. It has been shown in [15] that (1.1) holds in case $f$ and $g$ are the sum of trigonometric polynomials and $(2 n+6)$-times continuously differentiable functions with compact support. In [13] this result was extended to symbols $f$ and $g$ whose derivatives up to order $(4 n+6)$ are continuous and bounded. It was shown recently in [5] that the second equation of (1.1) even holds for bounded uniformly continuous symbols (BUC).

The goal of the present paper is to push this result even further by considering (no longer bounded) uniformly continuous functions $\left(\mathrm{UC}\left(\mathbb{C}^{n}\right)\right)$ as well the classical space [20] of bounded functions of vanishing mean oscillation $\left(\operatorname{VMO}\left(\mathbb{C}^{n}\right) \cap L^{\infty}\left(\mathbb{C}^{n}\right)\right)$. We note that the algebra $\operatorname{BUC}\left(\mathbb{C}^{n}\right)$ is contained in $\operatorname{VMO}\left(\mathbb{C}^{n}\right) \cap L^{\infty}\left(\mathbb{C}^{n}\right)$ but $\operatorname{VMO}\left(\mathbb{C}^{n}\right) \cap L^{\infty}\left(\mathbb{C}^{n}\right)$ is a well-studied algebra which also contains discontinuous functions.

The corresponding results for bounded symmetric domains have been obtained recently in [7] and the methods of proof are rather similar. Consider the standard identity

$$
T_{f}^{(t)} T_{g}^{(t)}-T_{f g}^{(t)}=-\left(H_{\bar{f}}^{(t)}\right)^{*} H_{g}^{(t)},
$$

where $H^{(t)}$ denotes the corresponding Hankel operator. The idea now is to show that $\left\|H_{\bar{f}}^{(t)}\right\|_{t}$ tends to 0 if $f$ is reasonably chosen. As a benefit of this approach, we only have to assume that $\left\|H_{g}^{(t)}\right\|_{t}$ is uniformly bounded near 0 in order to obtain

$$
\lim _{t \rightarrow 0}\left\|T_{f}^{(t)} T_{g}^{(t)}-T_{f g}^{(t)}\right\|_{t}=0 .
$$

In particular, $g$ can be chosen to be an arbitrary $L^{\infty}$-function.

Here is a short outline of our approach: In Section 2 we fix the notation. Let $f$ be a function of bounded mean oscillation. We generalize a norm estimate on Hankel operators in [2] to the family of Hankel operators $H_{f}^{(t)}, t>0$ acting on differently weighted Fock spaces. The main issue here is to choose the constant which appears in the norm estimate independently 
of the weight parameter $t$. Sections 3 and 4 contain the proof of the semi-classial limit $(*)$ for uniformly continuous operator symbols and bounded symbols in $\operatorname{VMO}\left(\mathbb{C}^{n}\right)$, respectively. In Section 5 of the paper we consider the algebra $\operatorname{Op}(L)$ of all decomposable bounded operators $X=\oplus_{t>0} X^{(t)}$ acting on the direct integral $L:=\int_{t>0}^{\oplus} L_{t}^{2}$ of standard Gaussian weighted $L^{2}$ spaces. An ideal $\mathcal{I} \subset \mathrm{Op}(L)$ is defined by

$$
\mathcal{I}:=\left\{X \in \mathrm{Op}(L): \lim _{t \rightarrow 0}\left\|X^{(t)}\right\|_{t}=0\right\} .
$$

Theorem 5.7 shows that the set $\mathcal{A}$ of all symbols $f \in L^{\infty}\left(\mathbb{C}^{n}\right)$ such that the semi-commutators

$$
T_{f} T_{g}-T_{f g} \quad \text { and } \quad T_{g} T_{f}-T_{g f}, \quad T_{g}:=\oplus_{t>0} T_{g}^{(t)}
$$

for all $g \in L^{\infty}\left(\mathbb{C}^{n}\right)$ belong to the ideal $\mathcal{I}$ is a closed and conjugate-closed subalgebra of $L^{\infty}\left(\mathbb{C}^{n}\right)$ and it precisely coincides with $\operatorname{VMO}\left(\mathbb{C}^{n}\right) \cap L^{\infty}\left(\mathbb{C}^{n}\right)$.

In Section 6 we show that the first equation of (1.1) holds for all $f \in L^{\infty}\left(\mathbb{C}^{n}\right)$. In Section 7 we provide some more examples of functions that do not satisfy the second equation of (1.1) (a first example was already given in [5]) as well as some further comments.

\section{Notation AND time DePEndent NORM ESTimates}

Let $n \in \mathbb{N}$ and consider the Euclidean $n$-space $\mathbb{C}^{n}$. With $z, w \in \mathbb{C}^{n}$ we denote by $\langle z, w\rangle:=$ $z_{1} \bar{w}_{1}+\cdots+z_{n} \bar{w}_{n}$ and $|z|=\sqrt{\langle z, z\rangle}$ the Euclidean inner product and norm, respectively. For $t>0$ we consider the following Gaussian probability measures

$$
d \mu_{t}(z)=\frac{1}{(4 \pi t)^{n}} e^{-\frac{|z|^{2}}{4 t}} d v(z)
$$

and the corresponding function space $L_{t}^{2}:=L^{2}\left(\mathbb{C}^{n}, d \mu_{t}\right)$ on $\mathbb{C}^{n}$. The closed subspace of analytic functions in $L_{t}^{2}$ is denoted by $H_{t}^{2}$ and it forms a Hilbert space with reproducing and normalized reproducing kernel

$$
K_{t}(z, w)=\exp \left\{\frac{\langle z, w\rangle}{4 t}\right\} \quad \text { and } \quad k_{w}^{t}(z)=\frac{K_{t}(z, w)}{\left\|K_{t}(\cdot, w)\right\|}=\exp \left\{\frac{\langle z, w\rangle}{4 t}-\frac{|w|^{2}}{8 t}\right\} .
$$

For a measurable function $f$ and an analytic function $g \in H_{t}^{2}$ with $f g \in L_{t}^{2}$, we write

$$
T_{f}^{(t)} g:=P^{(t)}(f g) \quad \text { and } \quad H_{f}^{(t)} g:=\left(I-P^{(t)}\right)(f g)
$$

for the corresponding Toeplitz and Hankel operators, respectively, where $P^{(t)}$ denotes the orthogonal projection from $L_{t}^{2}$ onto $H_{t}^{2}$. Note that $P^{(t)}$ can be expressed as the integral operator:

$$
\left[P^{(t)} h\right](z)=\int_{\mathbb{C}^{n}} h(u) K_{t}(z, u) d \mu_{t}(u), \quad h \in L_{t}^{2}
$$


On $L_{t}^{2}$ we have the usual inner product

$$
\langle f, g\rangle_{t}=\int_{\mathbb{C}^{n}} f \bar{g} d \mu_{t} \quad \text { with } \quad\|f\|_{t}^{2}=\langle f, f\rangle_{t} .
$$

For any linear operator $X$ with domain and range in $L_{t}^{2}$ we have the usual operator norm $\|X\|_{t}=\sup \left\{\|X f\|_{t} /\|f\|_{t}: f \neq 0\right\}$. For bounded (or essentially bounded) functions $f$ we write $\|f\|_{\infty}$ for the supremum (or essential supremum) of $|f|$.

In some of our results we allow operator symbols $f$ in the space $\mathrm{UC}\left(\mathbb{C}^{n}\right)$ which contains unbounded functions. Then the Toeplitz operator $T_{f}^{(t)}$ may be unbounded as well. Hence we have to specify the domain and carefully define operator products. This issue is discussed in [1] where a function space $\operatorname{Sym}\left(\mathbb{C}^{n}\right)$ and an increasing scale $\left(\mathcal{H}_{t}^{n}\right)_{n \in \mathbb{N}}$ of Hilbert spaces in $L_{t}^{2}$ are defined. It is shown that $P^{(t)}$ and the multiplication $M_{f}$ for all $f \in \operatorname{Sym}\left(\mathbb{C}^{n}\right)$ are operators acting on this scale. In particular, finite products of these operators are welldefined. One easily checks that $\operatorname{Sym}\left(\mathbb{C}^{n}\right)$ contains the space $\mathrm{UC}\left(\mathbb{C}^{n}\right)$ and therefore we can form finite products of Toeplitz operators with uniformly continuous symbols.

We will also need the heat transform

$$
\tilde{f}^{(t)}(w):=\frac{1}{(4 \pi t)^{n}} \int_{\mathbb{C}^{n}} f(w-z) e^{-\frac{|z|^{2}}{4 t}} d v(z)=\int_{\mathbb{C}^{n}} f(w-z) d \mu_{t}(z) .
$$

Remark 2.1. Note that $\tilde{f}^{(t)}(w)=\left\langle T_{f}^{(t)} k_{w}^{t}, k_{w}^{t}\right\rangle_{t}$ for $w \in \mathbb{C}^{n}$ so that $\left\|\tilde{f}^{(t)}\right\|_{\infty} \leq\left\|T_{f}^{(t)}\right\|_{t}$ by the Cauchy-Schwarz inequality.

The mean oscillation of a function $f$ is given by

$$
\operatorname{MO}^{t}(f)(w)=\left(|f|^{2}\right)^{\sim(t)}(w)-\left|\tilde{f}^{(t)}(w)\right|^{2}=\int_{\mathbb{C}^{n}}\left|f(w-z)-\tilde{f}^{(t)}(w)\right|^{2} d \mu_{t}(z) .
$$

We will say that a function $f$ has bounded mean oscillation if the semi-norm

$$
\|f\|_{\mathrm{BMO}_{*}^{t}}:=\sup _{w \in \mathbb{C}^{n}} \sqrt{\operatorname{MO}^{t}(f)(w)}
$$

is finite. The (linear) space of all functions having finite $\mathrm{BMO}_{*}^{t}$ semi-norm is denoted by

$$
\mathrm{BMO}_{*}\left(\mathbb{C}^{n}\right):=\left\{f \in L_{\mathrm{loc}}^{1}\left(\mathbb{C}^{n}\right):\|f\|_{\mathrm{BMO}_{*}^{t}}<\infty\right\} .
$$

Recall that the right hand side (as a vector space) does not depend on the parameter $t$ (see [6]) and therefore we do not indicate $t$ in the notation. A different, more standard version of $\mathrm{BMO}_{*}\left(\mathbb{C}^{n}\right)$ is considered in Section 4 . As is well-known $\mathrm{BMO}_{*}\left(\mathbb{C}^{n}\right)$ contains unbounded functions. Hankel operators with symbols in $\mathrm{BMO}_{*}\left(\mathbb{C}^{n}\right)$ are considered in [2] and it is shown that they are bounded on a dense domain. We mention that our notations of the spaces $\mathrm{BMO}_{*}\left(\mathbb{C}^{n}\right), \operatorname{BMO}\left(\mathbb{C}^{n}\right)$ and $\operatorname{VMO}\left(\mathbb{C}^{n}\right)$ in Section 4 are different from the ones in [2]. 
In the remaining part of this section we generalize a norm estimate for Hankel operators in [2] to operators acting on the above family of Hilbert spaces. For each $t>0$ consider the operator

$$
U_{t}: L_{t}^{2} \rightarrow L_{\frac{1}{4}}^{2}: f \mapsto\left[U_{t} f\right](z):=f(z 2 \sqrt{t}) .
$$

A simple calculation shows that $U_{t}$ is an isometry onto $L_{\frac{1}{4}}^{2}$ with inverse $U_{t}^{-1}=U_{\frac{1}{16 t}}$. Moreover, $U_{t}$ restricts to an isometry from $H_{t}^{2}$ onto $H_{\frac{1}{4}}^{2}$ and for all $f \in L^{\infty}\left(\mathbb{C}^{n}\right)$ one has:

$$
U_{t} M_{f}=M_{f(\cdot 2 \sqrt{t})} U_{t} \quad \text { and } \quad U_{t} P^{(t)}=P^{\left(\frac{1}{4}\right)} U_{t} .
$$

Clearly, the first equality remains true for an unbounded operator symbol $f$ when we restrict the product of operators to the maximal domain of $M_{f}$. Combining these two relations gives

$$
\begin{gathered}
U_{t} T_{f}^{(t)} U_{t}^{-1}=T_{f(\cdot 2 \sqrt{t})}^{\left(\frac{1}{4}\right)}, \\
U_{t} H_{f}^{(t)} U_{t}^{-1}=H_{f(\cdot 2 \sqrt{t})}^{\left(\frac{1}{4}\right)} .
\end{gathered}
$$

The following result is Theorem 4.2 in [2]:

Theorem 2.2. ([2]) Let $f \in \mathrm{BMO}_{*}\left(\mathbb{C}^{n}\right)$. Then the Hankel operator $H_{f}^{\left(\frac{1}{4}\right)}$ is bounded and there is a constant $C>0$ independent of $f$ such that

$$
\left\|H_{f}^{\left(\frac{1}{4}\right)}\right\|_{\frac{1}{4}} \leq C\|f\|_{\mathrm{BMO}_{*}^{\frac{1}{4}}} .
$$

We can use the family of isometries $\left(U_{t}\right)_{t>0}$ between the differently weighted $L^{2}$-spaces to generalize the inequality (2.7) with a $t$-independent constant $C$. Note that for $z \in \mathbb{C}^{n}$ :

$$
\{f(\cdot 2 \sqrt{t})\}^{\widetilde{(}^{\left(\frac{1}{4}\right)}}(z)=\tilde{f}^{(t)}(z 2 \sqrt{t})
$$

and therefore we obtain the identity:

$$
\|f(\cdot 2 \sqrt{t})\|_{\mathrm{BMO}_{*}^{\frac{1}{4}}}=\|f\|_{\mathrm{BMO}_{*}^{t}} .
$$

Corollary 2.3. Let $f \in \mathrm{BMO}_{*}\left(\mathbb{C}^{n}\right)$. Then there is a constant $C>0$ independent of $t>0$ and $f$ such that

$$
\left\|H_{f}^{(t)}\right\|_{t} \leq C\|f\|_{\mathrm{BMO}_{*}^{t}}
$$

Proof. We combine Theorem 2.2 with (2.6) and (2.8):

$$
\left\|H_{f}^{(t)}\right\|_{t}=\left\|U_{t} H_{f}^{(t)} U_{t}^{-1}\right\|_{\frac{1}{4}}=\left\|H_{f(\cdot 2 \sqrt{t})}^{\left(\frac{1}{4}\right)}\right\|_{\frac{1}{4}} \leq C\|f(\cdot 2 \sqrt{t})\|_{\mathrm{BMO}_{*}^{\frac{1}{4}}}=C\|f\|_{\mathrm{BMO}_{*}^{t}},
$$

where $C>0$ is the constant in Theorem 2.2 which is independent of $t$ and $f$. 


\section{OpERATORS WITH UNIFORMLY CONTINUOUS SYMBOLS}

In the following we denote by $\mathrm{UC}\left(\mathbb{C}^{n}\right)$ the space of all uniformly continuous complex valued functions on $\mathbb{C}^{n}$. Moreover, we write

$$
\mathrm{BUC}\left(\mathbb{C}^{n}\right)=\mathrm{UC}\left(\mathbb{C}^{n}\right) \cap L^{\infty}\left(\mathbb{C}^{n}\right)
$$

for the $C^{*}$ algebra of all bounded uniformly continuous functions. The next two propositions recall some results from [4]:

Proposition 3.1. Let $f \in \mathrm{UC}\left(\mathbb{C}^{n}\right)$. Then $f$ is in $\mathrm{BMO}_{*}\left(\mathbb{C}^{n}\right)$, $\tilde{f}^{(t)}$ is Lipschitz continuous and $f-\tilde{f}^{(t)}$ is bounded. Moreover, the Lipschitz constant of $\tilde{f}^{(t)}$ is bounded by $\frac{1}{\sqrt{t}}\|f\|_{\mathrm{BMO}_{*}^{t}}$.

Proof. See [4, Lemma 2.1] and [4, Proposition 3.1] for the first two statements. In order to estimate the Lipschitz constant of $\tilde{f}^{(t)}$ recall that in the proof of [4, Proposition 3.1] (see also [2, Corollary 2.7]) it was shown that for all $t>0$ :

$$
\left|\tilde{f}^{(t)}(z)-\tilde{f}^{(t)}(w)\right| \leq t^{-\frac{1}{2}}\|f(\cdot 2 \sqrt{t})\|_{\mathrm{BMO}_{*}^{\frac{1}{4}}}|z-w|=t^{-\frac{1}{2}}\|f\|_{\mathrm{BMO}_{*}^{t}}|z-w| .
$$

In the last equality we have use the identity (2.8) again.

Proposition 3.2. Let $f \in \mathrm{UC}\left(\mathbb{C}^{n}\right)$. Then $\tilde{f}^{(t)} \rightarrow f$ uniformly on $\mathbb{C}^{n}$.

Proof. See [4, Proposition 3.2].

Proposition 3.3. Let $f \in \mathrm{UC}\left(\mathbb{C}^{n}\right)$. Then $\|f\|_{\mathrm{BMO}_{*}^{t}} \rightarrow 0$ as $t \rightarrow 0$.

Proof. Let $\frac{1}{4}>\varepsilon>0$ be fixed and choose $\delta>0$ such that $|f(z)-f(w)|<\varepsilon$ for all $z, w \in \mathbb{C}^{n}$ with $|z-w|<\delta$. We divide the domain of integration into two parts:

$$
\operatorname{MO}^{t}(f)(w)=\left\{\int_{|z|<\delta}+\int_{|z| \geq \delta}\right\}\left|f(w-z)-\tilde{f}^{(t)}(w)\right|^{2} d \mu_{t}(z) .
$$

By Proposition 3.2, we have $\left\|f-\tilde{f}^{(t)}\right\|_{\infty} \leq \varepsilon$ for sufficiently small $t$. Thus the integrand can be estimated as follows:

$$
\begin{aligned}
\left|f(w-z)-\tilde{f}^{(t)}(w)\right|^{2} & \leq\left(|f(w-z)-f(w)|+\left|f(w)-\tilde{f}^{(t)}(w)\right|\right)^{2} \\
& \leq(|f(w-z)-f(w)|+\varepsilon)^{2}
\end{aligned}
$$

In the case $|z|<\delta$ we get $|f(w-z)-f(w)|<\varepsilon$ and thus

$$
\int_{|z|<\delta}\left|f(w-z)-\tilde{f}^{(t)}(w)\right|^{2} d \mu_{t}(z) \leq 4 \varepsilon^{2}<\varepsilon
$$

for sufficiently small $t$. 
In the case $|z| \geq \delta$ we observe that $f$ is the sum of a bounded and a Lipschitz continuous function (cf. Proposition 3.1) and hence $|f(w-z)-f(w)| \leq C(1+|z|$ ) for a suitable constant C. Now,

$$
\begin{aligned}
\int_{|z| \geq \delta}\left|f(w-z)-\tilde{f}^{(t)}(w)\right|^{2} d \mu_{t}(z) & \leq \frac{1}{(4 \pi t)^{n}} \int_{|z| \geq \delta}(C(1+|z|)+\varepsilon)^{2} e^{-\frac{|z|^{2}}{4 t}} d v(z) \\
& \leq \frac{1}{(4 \pi t)^{n}} e^{-\frac{\delta^{2}}{8 t}} \int_{|z| \geq \delta}(C(1+|z|)+\varepsilon)^{2} e^{-|z|^{2}} d v(z)
\end{aligned}
$$

for $t \leq \frac{1}{8}$. As the integral on the right-hand side is bounded, the whole term converges to 0 as $t \rightarrow 0$. We conclude $\mathrm{MO}^{t}(f)(w) \rightarrow 0$ uniformly in $w$. Thus the assertion follows.

Theorem 3.4. Let $f \in \mathrm{UC}\left(\mathbb{C}^{n}\right)$. Then $\lim _{t \rightarrow 0}\left\|H_{f}^{(t)}\right\|_{t}=0$. In particular,

$$
\lim _{t \rightarrow 0}\left\|T_{f}^{(t)} T_{g}^{(t)}-T_{f g}^{(t)}\right\|_{t}=0
$$

for all $g \in L^{\infty}\left(\mathbb{C}^{n}\right)$ or all $g \in \mathrm{UC}\left(\mathbb{C}^{n}\right)$.

Proof. Because of $T_{f}^{(t)} T_{g}^{(t)}-T_{f g}^{(t)}=-\left(H_{\bar{f}}^{(t)}\right)^{*} H_{g}^{(t)}$, it is sufficient to show

$$
\lim _{t \rightarrow 0}\left\|H_{f}^{(t)}\right\|_{t}=0
$$

This follows from Corollary 2.3 in combination with Proposition 3.3 .

Another interesting question is: For which symbols $f$ and $g$ are the semi-commutators $T_{f}^{(t)} T_{g}^{(t)}-T_{f g}^{(t)}$ in (3.3) compact? In other words, what is the maximal algebra $\mathcal{T}$ generated by Toeplitz operators so that the Calkin algebra $\mathcal{T} / \mathcal{K}\left(H_{t}^{2}\right)$ is commutative? For BUCsymbols we will answer these questions in Proposition 3.6 below. Therefore we need the following notions:

For a bounded and continuous function $f: \mathbb{C}^{n} \rightarrow \mathbb{C}$ (in short: $f \in \mathrm{BC}\left(\mathbb{C}^{n}\right)$ ) define

$$
\mathrm{Osc}_{z}(f):=\sup \{|f(z)-f(w)|:|z-w|<1\} .
$$

The space of functions having vanishing oscillation is

$$
\mathrm{VO}\left(\mathbb{C}^{n}\right):=\left\{f \in \mathrm{BC}\left(\mathbb{C}^{n}\right): \operatorname{Osc}_{z}(f) \rightarrow 0 \text { as }|z| \rightarrow \infty\right\}
$$

Note that in fact $\operatorname{VO}\left(\mathbb{C}^{n}\right) \subset \operatorname{BUC}\left(\mathbb{C}^{n}\right)$. Indeed, for $f \in \operatorname{VO}\left(\mathbb{C}^{n}\right)$ and $\varepsilon>0$ we may choose a compact subset $K \subset \mathbb{C}^{n}$ such that $\operatorname{Osc}_{z}(f)<\varepsilon$ for all $z \in \mathbb{C}^{n} \backslash K$. As $f$ is uniformly continuous on compact sets, there is a $\delta_{K}$ so that $|f(z)-f(w)|<\varepsilon$ for all $z \in K, w \in \mathbb{C}^{n}$ with $|z-w|<\delta_{K}$. Thus $|f(z)-f(w)|<\varepsilon$ for all $z, w \in \mathbb{C}^{n}$ with $|z-w|<\min \left\{\delta_{K}, 1\right\}$.

Here is one possible way of constructing functions in $\operatorname{VO}\left(\mathbb{C}^{n}\right)$ : 
ExAmple 3.5. Choose $f \in \mathrm{BUC}(\mathbb{R})$ and compose it with a function $g: \mathbb{C}^{n} \rightarrow \mathbb{R}$ that is continuous on $\mathbb{C}^{n}$, differentiable in a neighborhood of $\infty$ and its derivative $g^{\prime}$ tends to 0 as $|z| \rightarrow \infty$. To show that $f \circ g$ is in $\operatorname{VO}\left(\mathbb{C}^{n}\right)$, choose $\varepsilon>0$ and select $\delta>0$ such that $|f(x)-f(y)|<\varepsilon$ for $x, y \in \mathbb{R}$ with $|x-y|<\delta$. By the mean value theorem we may choose $R>0$ sufficiently large such that $|g(z)-g(w)|<\delta$ for all $z, w \in \mathbb{C}^{n}$ with $|z|>R$ and $|z-w|<1$. This implies $|(f \circ g)(z)-(f \circ g)(w)|<\varepsilon$ and hence $\operatorname{Osc}_{z}(f \circ g) \rightarrow 0$ as $|z| \rightarrow \infty$. We conclude $f \circ g \in \operatorname{VO}\left(\mathbb{C}^{n}\right)$.

For a concrete example consider $z \mapsto \exp (i \sqrt{|z|})$.

With these preparations we can now prove the following proposition.

Proposition 3.6. Let $f \in \mathrm{BUC}\left(\mathbb{C}^{n}\right)$ and $t>0$. Then the following are equivalent:

(i) $f \in \operatorname{VO}\left(\mathbb{C}^{n}\right)$,

(ii) $H_{f}^{(t)}$ and $H_{\bar{f}}^{(t)}$ are compact,

(iii) $T_{f}^{(t)} T_{g}^{(t)}-T_{f g}^{(t)}$ and $T_{g}^{(t)} T_{f}^{(t)}-T_{g f}^{(t)}$ are compact for all $g \in L^{\infty}\left(\mathbb{C}^{n}\right)$.

Proof. Let $f \in \operatorname{VO}\left(\mathbb{C}^{n}\right)$. Then $H_{f}^{(t)}$ and $H_{\bar{f}}^{(t)}$ are compact by a straight forward extension of [2, Theorem 5.3] from the case $t=\frac{1}{4}$ to general $t>0$.

Now assume that $H_{f}^{(t)}$ and $H_{\bar{f}}^{(t)}$ are both compact. Then $\tilde{f}^{(t)} \in \operatorname{VO}\left(\mathbb{C}^{n}\right)$ by $[2$, Theorem 5.3] again and $T_{f-\tilde{f}^{(t)}}^{(t)}$ is compact by [2, Theorem 3.1]. Moreover, $f-\tilde{f}^{(t)} \in \mathrm{BUC}\left(\mathbb{C}^{n}\right)$ and thus $f-\tilde{f}^{(t)} \in C_{0}\left(\mathbb{C}^{n}\right) \subset \operatorname{VO}\left(\mathbb{C}^{n}\right)$ by [3, Theorem 2.3]. As the sum of two functions in $\operatorname{VO}\left(\mathbb{C}^{n}\right)$ is obviously again in $\operatorname{VO}\left(\mathbb{C}^{n}\right)$, we conclude that $f$ is in $\operatorname{VO}\left(\mathbb{C}^{n}\right)$.

That (ii) and (iii) are equivalent is standard.

\section{Symbols IN VMO}

In the present section we consider the quantization problem for operators with symbols of vanishing mean oscillation. We start by recalling some notation. Consider locally integrable functions $f: \mathbb{R}^{n} \rightarrow \mathbb{C}$ with average value

$$
f_{E}=\frac{1}{|E|} \int_{E} f
$$

on a bounded measurable subset $E \subset \mathbb{R}^{n}$ with finite measure $|E|$. We consider the variance of $f$ on $E$

$$
\operatorname{Var}_{E}(f)=\frac{1}{|E|} \int_{E}\left|f-f_{E}\right|^{2}
$$

as well as the corresponding quantity

$$
\operatorname{Osc}_{E}(f)=\frac{1}{|E|} \int_{E}\left|f-f_{E}\right| .
$$


Definition 4.1. (see [20, 21]) We say $f$ is in $\mathrm{BMO}\left(\mathbb{R}^{n}\right)$ if the set

$$
\left\{\operatorname{Osc}_{E}(f): \text { E each } n \text {-cube in } \mathbb{R}^{n}\right\}
$$

is bounded. We say $f$ is in $\operatorname{VMO}\left(\mathbb{R}^{n}\right)$ if $f$ is in $\operatorname{BMO}\left(\mathbb{R}^{n}\right)$ and, for all $n$-cubes $E$,

$$
\lim _{a \rightarrow 0} \sup \left\{\operatorname{Osc}_{E}(f):|E| \leq a\right\}=0 \text {. }
$$

If we replace $\operatorname{Osc}_{E}(f)$ by $\operatorname{Var}_{E}(f)$, we get new sets $\mathrm{BMO}_{2}\left(\mathbb{R}^{n}\right)$ and $\mathrm{VMO}_{2}\left(\mathbb{R}^{n}\right)$.

Remark 4.2. $\mathrm{BMO}\left(\mathbb{R}^{2 n}\right)$ and $\mathrm{BMO}_{*}\left(\mathbb{C}^{n}\right)$ defined earlier are quite different. In particular, $\mathrm{UC}\left(\mathbb{R}^{2 n}\right) \cong \mathrm{UC}\left(\mathbb{C}^{n}\right)$ is not contained in $\mathrm{BMO}\left(\mathbb{R}^{2 n}\right)$.

The Cauchy-Schwarz inequality shows that

$$
\mathrm{BMO}_{2}\left(\mathbb{R}^{n}\right) \subset \operatorname{BMO}\left(\mathbb{R}^{n}\right), \quad \operatorname{VMO}_{2}\left(\mathbb{R}^{n}\right) \subset \operatorname{VMO}\left(\mathbb{R}^{n}\right) .
$$

Direct calculation shows (eg. [8, p. 313])

Lemma 4.3. We have, for arbitrary bounded measurable subsets E,

$$
\operatorname{Var}_{E}(f)=\frac{1}{2|E|^{2}} \int_{E} \int_{E}|f(z)-f(w)|^{2} d v(z) d v(w)
$$

so that, for $F \subset E$,

$$
\operatorname{Var}_{E}(f) \geq \frac{|F|^{2}}{|E|^{2}} \operatorname{Var}_{F}(f)
$$

Remark 4.4. Because of Lemma 4.3, $\mathrm{BMO}_{2}\left(\mathbb{R}^{n}\right)$ and $\mathrm{VMO}_{2}\left(\mathbb{R}^{n}\right)$ have the additional property that $n$-cubes can be replaced by $n$-balls in their definitions. We need only consider the inscribed and circumscribed balls for a given cube.

In the analysis that follows, we identify $\mathbb{C}^{n}$ with $\mathbb{R}^{2 n}$ in the standard way and we consider the set $\operatorname{VMO}\left(\mathbb{C}^{n}\right) \cap L^{\infty}\left(\mathbb{C}^{n}\right)$, the essentially bounded functions in $\operatorname{VMO}\left(\mathbb{C}^{n}\right)$. It is easy to see that $\mathrm{BUC}\left(\mathbb{C}^{n}\right) \subset \mathrm{VMO}_{2}\left(\mathbb{C}^{n}\right)$. However, there are discontinuous functions in $\mathrm{VMO}\left(\mathbb{C}^{n}\right) \cap$ $L^{\infty}\left(\mathbb{C}^{n}\right)$, [21, p.290]. We have:

Theorem 4.5. $\operatorname{VMO}\left(\mathbb{C}^{n}\right) \cap L^{\infty}\left(\mathbb{C}^{n}\right)$ is a sup norm-closed, conjugate closed subalgebra of $L^{\infty}\left(\mathbb{C}^{n}\right)$ with

$$
\operatorname{VMO}\left(\mathbb{C}^{n}\right) \cap L^{\infty}\left(\mathbb{C}^{n}\right)=\mathrm{VMO}_{2}\left(\mathbb{C}^{n}\right) \cap L^{\infty}\left(\mathbb{C}^{n}\right)
$$

Proof. A easy estimate using $\left|f-f_{E}\right| \leq 2\|f\|_{\infty}$ implies (4.1). Integrating the inequality

$$
|f(z) g(z)-f(w) g(w)|^{2} \leq 2\|f\|_{\infty}^{2}|g(z)-g(w)|^{2}+2\|g\|_{\infty}^{2}|f(z)-f(w)|^{2}
$$

over $E \times E$ shows that for $f, g$ in $\mathrm{VMO}_{2}\left(\mathbb{C}^{n}\right) \cap L^{\infty}\left(\mathbb{C}^{n}\right)$, we also have $f g$ in $\mathrm{VMO}_{2}\left(\mathbb{C}^{n}\right) \cap$ $L^{\infty}\left(\mathbb{C}^{n}\right)$. The remainder of the proof is standard.

Remark 4.6. Theorem 4.5 gives, for $\mathbb{C}^{n}$ (or $\mathbb{R}^{n}$ ), a classical result of Sarason [20] for the circle. 
Now we consider the quantization problem for Toeplitz operators with bounded symbols in $\operatorname{VMO}\left(\mathbb{C}^{n}\right)$. In the definitions above and for convenience we pass from $n$-cubes $E \subset \mathbb{C}^{n}$ to Euclidean $n$-balls

$$
B(x, \rho):=\left\{z \in \mathbb{C}^{n}:|z-x|<\rho\right\}
$$

centered at $x \in \mathbb{C}^{n}$ and with radius $\rho>0$, cf. Remark 4.4.

Lemma 4.7. Let $f \in L^{\infty}\left(\mathbb{C}^{n}\right)$ and $x \in \mathbb{C}^{n}$. Then, for all $\rho>0$ and all $t>0$ we have

$$
\operatorname{MO}^{t}(f)(x) \leq \int_{\mathbb{C}^{n}}\left|f(x-z)-f_{B(x, \rho)}\right|^{2} d \mu_{t}(z) .
$$

Proof. Consider the function $L: \mathbb{C} \rightarrow[0, \infty)$ defined by

$$
L(c)=\int_{\mathbb{C}^{n}}|f(x-z)-c|^{2} d \mu_{t}(z) .
$$

Standard arguments show that $L$ attains a global minimum at $c=\tilde{f}^{(t)}(x)$. Hence the lemma follows from $\mathrm{MO}^{t}(f)(x)=L \circ \tilde{f}^{(t)}(x)$.

Theorem 4.8. Let $f \in \operatorname{VMO}\left(\mathbb{C}^{n}\right) \cap L^{\infty}\left(\mathbb{C}^{n}\right)$, then $\lim _{t \rightarrow 0}\|f\|_{\mathrm{BMO}_{*}^{t}}=0$.

Proof. Let $t>0$ and fix a parameter $\alpha>0$ which we will specify later on. According to Lemma 4.7 we can estimate the mean oscillation of $f$ as follows:

$$
\mathrm{MO}^{t}(f)(x) \leq\left\{\int_{|z| \leq \alpha \sqrt{t}}+\int_{|z|>\alpha \sqrt{t}}\right\}\left|f(x-z)-f_{B(x, \alpha \sqrt{t})}\right|^{2} d \mu_{t}(z) .
$$

We denote the first and the second integral by $I_{1, t, \alpha}(x)$ and $I_{2, t, \alpha}(x)$, respectively, and estimate them separately. By the Cauchy-Schwarz inequality we have

$$
\begin{aligned}
I_{1, t, \alpha}(x)= & \frac{1}{(4 \pi t)^{n}} \int_{|x-z| \leq \alpha \sqrt{t}}\left|f(z)-f_{B(x, \alpha \sqrt{t})}\right|^{2} e^{-\frac{|x-z|^{2}}{4 t}} d v(z) \\
& \leq \frac{1}{(4 \pi t)^{n}}\left\{\int_{B(x, \alpha \sqrt{t})} e^{-\frac{|x-z|^{2}}{2 t}} d v(z)\right\}^{\frac{1}{2}}\left\{\int_{B(x, \alpha \sqrt{t})}\left|f(z)-f_{B(x, \alpha \sqrt{t})}\right|^{4} d v(z)\right\}^{\frac{1}{2}} .
\end{aligned}
$$

The first integral takes the form

$$
\int_{B(x, \alpha \sqrt{t})} e^{-\frac{|x-z|^{2}}{2 t}} d v(z)=(2 t)^{n} C_{\alpha} \quad \text { where } \quad C_{\alpha}=\int_{B(0, \alpha)} e^{-|z|^{2}} d v(z) .
$$

Therefore

$$
I_{1, t, \alpha}(x) \leq 2\|f\|_{\infty} \frac{\sqrt{C_{\alpha}}}{\left(8 \pi^{2} t\right)^{\frac{n}{2}}} \sqrt{|B(x, \alpha \sqrt{t})|} \cdot \sqrt{\operatorname{Var}_{B(x, \alpha \sqrt{t})}(f)} .
$$


Since $t^{-\frac{n}{2}} \sqrt{|B(x, \alpha \sqrt{t})|}=O(1)$ as $t \rightarrow 0$ and $\lim _{t \rightarrow 0} \sup \left\{\operatorname{Var}_{B(x, \alpha \sqrt{t})}(f): x \in \mathbb{C}^{n}\right\}=0$ it follows that

$$
\lim _{t \rightarrow 0} I_{1, t, \alpha}(x)=0
$$

uniformly for $x \in \mathbb{C}^{n}$. Next we estimate the second integral $I_{2, t, \alpha}(x)$. We obtain

$$
I_{2, t, \alpha}(x) \leq 4\|f\|_{\infty}^{2} \int_{|z|>\alpha \sqrt{t}} d \mu_{t}(z)=\frac{4\|f\|_{\infty}^{2}}{(4 \pi)^{n}} \int_{|z|>\alpha} e^{-\frac{|z|^{2}}{4}} d v(z) .
$$

Let $\varepsilon>0$ and with (4.3) choose $\alpha>0$ sufficiently large such that $0 \leq I_{2, t, \alpha}(x)<\varepsilon$. With this fixed $\alpha>0$ and (4.2) we can choose $t>0$ sufficiently small such that $I_{1, t, \alpha}(x)<\varepsilon$ for all $x \in \mathbb{C}^{n}$. Then

$$
\left\|\mathrm{MO}^{t}(f)\right\|_{\infty}<2 \varepsilon
$$

and the assertion follows.

Theorem 4.9. Let $f \in \operatorname{VMO}\left(\mathbb{C}^{n}\right) \cap L^{\infty}\left(\mathbb{C}^{n}\right)$, then $\lim _{t \rightarrow 0}\left\|H_{f}^{(t)}\right\|_{t}=0$ and, in particular, we have

$$
\lim _{t \rightarrow 0}\left\|T_{f}^{(t)} T_{g}^{(t)}-T_{f g}^{(t)}\right\|_{t}=0
$$

for all $g \in L^{\infty}\left(\mathbb{C}^{n}\right)$ and all $g \in \mathrm{UC}\left(\mathbb{C}^{n}\right)$.

Proof. It is sufficient to show that $\lim _{t \rightarrow 0}\left\|H_{f}^{(t)}\right\|_{t}=0$. In fact, this follows from Theorem 4.8 and Corollary 2.3 .

\section{Fock Quantization Algebras}

We now consider the direct integral $L:=\int_{\mathbb{R}_{+}}^{\oplus} L_{t}^{2}$ of $L_{t}^{2}$-spaces. In the following we write decomposable operators $X$ on $L$ in the form

$$
X=\oplus_{t>0} X^{(t)} \quad \text { for } \quad X^{(t)} \in \mathcal{L}\left(L_{t}^{2}\right) \quad \text { with } \quad\|X\|:=\sup _{t>0}\left\|X^{(t)}\right\|<\infty .
$$

The algebra of such operators is denoted by $\mathrm{Op}(L)$. The set

$$
\mathcal{I}:=\left\{X \in \mathrm{Op}(L): \lim _{t \rightarrow 0}\left\|X^{(t)}\right\|_{t}=0\right\}
$$

is a closed two-sided ideal in $\operatorname{Op}(L)$. We also consider the direct integral of the Fock spaces

$$
H:=\int_{\mathbb{R}_{+}}^{\oplus} H_{t}^{2}
$$

Remark 5.1. The Toeplitz and Hankel operators $T_{f}=\oplus_{t>0} T_{f}^{(t)}$ and $H_{f}=\oplus_{t>0} H_{f}^{(t)}$ are in $\operatorname{Op}(L)$ for $f \in L^{\infty}$. The Toeplitz operators are also in $\operatorname{Op}(H)$. 
Noting that

$$
T_{f}^{(t)} T_{g}^{(t)}-T_{f g}^{(t)}=-\left(H_{\bar{f}}^{(t)}\right)^{*} H_{g}^{(t)}
$$

for all $t>0$, we consider the set

$$
\mathcal{A}:=\left\{f \in L^{\infty}\left(\mathbb{C}^{n}\right): T_{f} T_{g}-T_{f g} \text { and } T_{g} T_{f}-T_{f g} \in \mathcal{I} \text { for all } g \in L^{\infty}\left(\mathbb{C}^{n}\right)\right\} .
$$

Proposition 5.2. $\mathcal{A}$ is a closed, conjugate-closed subalgebra of $L^{\infty}\left(\mathbb{C}^{n}\right)$ and we have

$$
\mathcal{A}=\left\{f \in L^{\infty}\left(\mathbb{C}^{n}\right): H_{f}, H_{\bar{f}} \in \mathcal{I}\right\} .
$$

Proof. For $\left\{f_{n}\right\} \subset \mathcal{A}$, with $f_{n} \rightarrow f \in L^{\infty}\left(\mathbb{C}^{n}\right)$, it is easy to check that $f \in \mathcal{A}$. For $f, h \in \mathcal{A}$ and $g \in L^{\infty}\left(\mathbb{C}^{n}\right)$, we have

$$
T_{f h} T_{g}-T_{f h g} \in T_{f} T_{h} T_{g}-T_{f h g}+\mathcal{I} \subset T_{f} T_{h g}-T_{f(h g)}+\mathcal{I} \subset \mathcal{I} .
$$

Hence $\mathcal{A}$ is an algebra, which clearly is invariant under complex conjugation of its elements. The equality of sets in (5.5) follows from (5.4).

Note that the definition of the algebra $\mathcal{A}$ and its characterization in (5.5) is given in terms of operator conditions. We now give an equivalent description which only involves a condition on the level of functions. Consider the set

$$
\mathcal{B}:=\left\{f \in L^{\infty}\left(\mathbb{C}^{n}\right): \lim _{t \rightarrow 0}\|f\|_{\mathrm{BMO}_{*}^{t}}=0\right\} .
$$

Proposition 5.3. $\mathcal{A}=\mathcal{B}$. In particular, $\mathcal{B}$ is a closed, conjugate-closed subalgebra of $L^{\infty}$.

Remark 5.4. One may check directly from the definition of $\mathcal{B}$, that it is a closed, conjugateclosed sub-algebra of $L^{\infty}\left(\mathbb{C}^{n}\right)$.

The next lemma serves as a preparation for the proof of Proposition 5.3. It generalizes (with the same proof) Lemma 2.5 in [2] to the family of weighted spaces $L_{t}^{2}$ and $H_{t}^{2}, t>0$.

Lemma 5.5. Let $t>0$ and $f \in L^{\infty}\left(\mathbb{C}^{n}\right)$. Then the mean oscillation of $f$ can be represented in the form

$$
\mathrm{MO}^{(t)}(f)(w)=\left\|\left(I-P^{(t)}\right)\left(f \circ \tau_{w}\right)\right\|_{t}^{2}+\left\|P^{(t)}\left[f \circ \tau_{w}\right]-\tilde{f}^{(t)}(w)\right\|_{t}^{2},
$$

where $\tau_{w}(z):=w-z$ for $z, w \in \mathbb{C}^{n}$ denotes the translation by $w$. Moreover,

$$
\|f\|_{\mathrm{BMO}_{*}^{t}} \leq \sqrt{\left\|H_{f}^{(t)}\right\|_{t}^{2}+\left\|H_{\frac{f}{f}}^{(t)}\right\|_{t}^{2}} .
$$

Proof. Let $w \in \mathbb{C}^{n}$ be fixed and note that $\left\langle P^{(t)}\left(f \circ \tau_{w}\right), \tilde{f}^{(t)}(w)\right\rangle_{t}=\left|\tilde{f}^{(t)}(w)\right|^{2}$. Therefore

$$
\left\|P^{(t)}\left[f \circ \tau_{w}\right]-\tilde{f}^{(t)}(w)\right\|_{t}^{2}=\left\|P^{(t)}\left[f \circ \tau_{w}\right]\right\|_{t}^{2}-\left|\tilde{f}^{(t)}(w)\right|^{2} .
$$

Using this relation and (2.2) we can express the mean oscillation in the form (5.7):

$$
\mathrm{MO}^{(t)}(f)(w)=\left\|f \circ \tau_{w}\right\|_{t}^{2}-\left|\tilde{f}^{(t)}(w)\right|^{2}
$$




$$
\begin{aligned}
& =\left\|\left(I-P^{(t)}\right)\left[f \circ \tau_{w}\right]\right\|_{t}^{2}+\left\|P^{(t)}\left[f \circ \tau_{w}\right]\right\|_{t}^{2}-\left|\tilde{f}^{(t)}(w)\right|^{2} \\
& =\left\|\left(I-P^{(t)}\right)\left[f \circ \tau_{w}\right]\right\|_{t}^{2}+\left\|P^{(t)}\left[f \circ \tau_{w}\right]-\tilde{f}^{(t)}(w)\right\|_{t}^{2}
\end{aligned}
$$

Now we can prove the estimate (5.8). Note that (2.1) implies:

$$
\begin{aligned}
\tilde{f}^{(t)}(w)=\left\langle f \circ \tau_{w}, 1\right\rangle_{t} & =\overline{\left\langle\bar{f} \circ \tau_{w}, K_{t}(\cdot, 0)\right\rangle_{t}}= \\
& =\overline{P^{(t)}\left(\bar{f} \circ \tau_{w}\right)(0)}=P^{(t)}\left(\overline{P^{(t)}\left(\bar{f} \circ \tau_{w}\right)}\right) .
\end{aligned}
$$

It follows:

$$
\begin{aligned}
\left\|P^{(t)}\left[f \circ \tau_{w}\right]-\tilde{f}^{(t)}(w)\right\|_{t}^{2} & =\left\|P^{(t)}\left[f \circ \tau_{w}\right]-P^{(t)}\left(\overline{P^{(t)}\left(\bar{f} \circ \tau_{w}\right)}\right)\right\|_{t}^{2} \\
& \leq\left\|f \circ \tau_{w}-\overline{P^{(t)}\left(\bar{f} \circ \tau_{w}\right)}\right\|_{t}^{2}=\left\|\left(I-P^{(t)}\right)\left(\bar{f} \circ \tau_{w}\right)\right\|_{t}^{2} .
\end{aligned}
$$

Together with (5.7) one obtains:

$$
\begin{aligned}
\mathrm{MO}^{(t)}(f)(w) & \leq\left\|\left(I-P^{(t)}\right)\left[f \circ \tau_{w}\right]\right\|_{t}^{2}+\left\|\left(I-P^{(t)}\right)\left[\bar{f} \circ \tau_{w}\right]\right\|_{t}^{2} \\
& =\left\|H_{f}^{(t)} k_{w}^{t}\right\|_{t}^{2}+\left\|H_{\bar{f}}^{(t)} k_{w}^{t}\right\|_{t}^{2} \leq\left\|H_{f}^{(t)}\right\|_{t}^{2}+\left\|H_{\bar{f}}^{(t)}\right\|_{t}^{2} .
\end{aligned}
$$

Now, (5.8) follows from the definition of the $\mathrm{BMO}_{*}^{t}$-semi-norm.

Proof. (Proposition 5.3). The inclusion $\mathcal{B} \subset \mathcal{A}$ directly follows from Corollary 2.3 whereas $\mathcal{A} \subset \mathcal{B}$ is a consequence of (5.5) in Proposition 5.2 and (5.8) in Lemma 5.5.

Note that, by Theorem 4.5 the space $\operatorname{VMO}\left(\mathbb{C}^{n}\right) \cap L^{\infty}\left(\mathbb{C}^{n}\right)$ is a closed, conjugate-closed subalgebra of $L^{\infty}\left(\mathbb{C}^{n}\right)$. From Theorem 4.8 we know that

$$
\operatorname{VMO}\left(\mathbb{C}^{n}\right) \cap L^{\infty}\left(\mathbb{C}^{n}\right) \subset \mathcal{B}=\mathcal{A}
$$

Lemma 5.6. There is $C_{n}>0$ only depending on the dimension $n$ such that for all $a \in \mathbb{C}^{n}$ :

$$
\|f\|_{\mathrm{BMO}_{*}^{t}}^{2} \geq \operatorname{MO}^{t}(f)(a) \geq C_{n} \operatorname{Var}_{B(a, \sqrt{t})}(f) .
$$

In particular, the following inclusion holds: $\mathcal{B} \subset \operatorname{VMO}\left(\mathbb{C}^{n}\right) \cap L^{\infty}\left(\mathbb{C}^{n}\right)$.

Proof. It is easy to check (see [8]) that

$$
\operatorname{MO}^{t}(f)(a)=\frac{1}{2} \int_{\mathbb{C}^{n}} \int_{\mathbb{C}^{n}}|f(u)-f(w)|^{2} d \mu_{t, a}(u) d \mu_{t, a}(w),
$$

where for $a, u \in \mathbb{C}^{n}$ we define:

$$
d \mu_{t, a}(u):=\left|k_{a}^{t}(u)\right|^{2} d \mu_{t}(u)=\frac{1}{(4 \pi t)^{n}} e^{-\frac{1}{4 t}|a-u|^{2}} d v(u) .
$$

Hence, by applying Lemma 4.3, we can estimate the mean oscillation from below as follows:

$$
\operatorname{MO}^{t}(f)(a) \geq \frac{1}{2} \frac{1}{(4 \pi t)^{2 n}} \int_{B(a, \sqrt{t})} \int_{B(a, \sqrt{t})}|f(u)-f(w)|^{2} e^{-\frac{|a-u|^{2}+|a-w|^{2}}{4 t}} d v(u) d v(w)
$$




$$
\begin{aligned}
& \geq \frac{e^{-\frac{1}{2}}}{(4 \pi t)^{2 n}} \cdot \frac{1}{2} \int_{B(a, \sqrt{t})} \int_{B(a, \sqrt{t})}|f(u)-f(w)|^{2} d v(u) d v(w) \\
& =e^{-\frac{1}{2} \frac{|B(a, \sqrt{t})|^{2}}{(4 \pi t)^{2 n}} \operatorname{Var}_{B(a, \sqrt{t})}(f)}
\end{aligned}
$$

Note that there is a constant $C_{n}$ only depending on the complex dimension $n$ such that

$$
e^{-\frac{1}{2}} \frac{|B(a, \sqrt{t})|^{2}}{(4 \pi t)^{2 n}}=C_{n}
$$

From this, the statement follows.

Finally, (5.9) together with Lemma 5.6 shows:

Theorem 5.7. $\mathcal{A}=\mathcal{B}=\operatorname{VMO}\left(\mathbb{C}^{n}\right) \cap L^{\infty}\left(\mathbb{C}^{n}\right)$.

Theorem 5.7 indicates that in case of the ideal $\mathcal{I}$ the algebra $\operatorname{VMO}\left(\mathbb{C}^{n}\right) \cap L^{\infty}\left(\mathbb{C}^{n}\right)$ plays a similar role as $\operatorname{VO}\left(\mathbb{C}^{n}\right)$ in case of compact operators (cf. Proposition [3.6).

\section{On The Limit of THE NORM OF TOEPLitz OPERATORS}

For each function $f$ in $\mathrm{BUC}\left(\mathbb{C}^{n}\right),[15$, Theorem 1] shows the following identity

$$
\lim _{t \rightarrow 0}\left\|T_{f}^{(t)}\right\|_{t}=\|f\|_{\infty} .
$$

Here, we extend this result to operator symbols $f \in L^{\infty}\left(\mathbb{C}^{n}\right)$ by showing that $\tilde{f}^{(t)}$ converges pointwise almost everywhere to $f$. The result will then be a consequence of Remark 2.1.

Recall that the heat transform $\left(\tilde{f}^{(t)}\right)_{t>0}$ has the semi-group property, i.e. for $s, t>0$ and $f \in L^{\infty}\left(\mathbb{C}^{n}\right)$ it holds

$$
\left\{\tilde{f}^{(t)} \widetilde{\}}^{(s)}=\widetilde{f}^{(s+t)} .\right.
$$

Moreover, the assignment $f \mapsto \tilde{f}^{(t)}$ is a contraction on $L^{\infty}\left(\mathbb{C}^{n}\right)$, i.e. $\left\|\tilde{f}^{(t)}\right\|_{\infty} \leq\|f\|_{\infty}$ for every $t>0$. Letting $s>t>0$ and combining these properties shows

$$
\left\|\tilde{f}^{(s)}\right\|_{\infty}=\|\left\{\tilde{f}^{(t)} \widetilde{\tau}^{(s-t)}\left\|_{\infty} \leq\right\| \tilde{f}^{(t)} \|_{\infty} .\right.
$$

Therefore $t \mapsto\left\|\tilde{f}^{(t)}\right\|_{\infty}$ is monotone decreasing and $\lim _{t \rightarrow 0}\left\|\tilde{f}^{(t)}\right\|_{\infty}$ exists for all $f \in L^{\infty}\left(\mathbb{C}^{n}\right)$.

As a further preparation, we show that the heat transform is bounded by the HardyLittlewood maximal function for any locally integrable $f$. The Hardy-Littlewood maximal function $f^{*}$ is defined by

$$
f^{*}(w):=\sup _{r>0} \frac{1}{|B(0, r)|} \int_{B(0, r)}|f(w-z)| d v(z),
$$

where $|B(0, r)|$ denotes the (Lebesgue) volume of the ball with radius $r$ as usual. 
Lemma 6.1. For every $t>0, w \in \mathbb{C}^{n}$ and any locally integrable function $f$ we have $\left|\tilde{f}^{(t)}(w)\right| \leq C f^{*}(w)$, where $C$ is some constant that only depends on the dimension $n$.

Proof. Recall that the volume of a (real) $2 n$-dimensional ball of radius $r$ is given by $\frac{\pi^{n}}{n !} r^{2 n}$. The result now follows by the following computation:

$$
\begin{aligned}
\left|\tilde{f}^{(t)}(w)\right| \leq & \frac{1}{(4 \pi t)^{n}} \int_{\mathbb{C}^{n}}|f(w-z)| e^{-\frac{|z|^{2}}{4 t}} d v(z) \\
\leq & \frac{1}{(4 \pi t)^{n}} \int_{B(0, \sqrt{4 t})}|f(w-z)| d v(z)+\frac{1}{(4 \pi t)^{n}} \int_{B(0, \sqrt{8 t}) \backslash B(0, \sqrt{4 t})}|f(w-z)| e^{-1} d v(z) \\
& +\ldots \\
\leq & \sum_{k=1}^{\infty} \frac{k^{n}}{n !} \frac{1}{|B(0, \sqrt{4 k t})|} \int_{B(0, \sqrt{4 k t})}|f(w-z)| e^{-(k-1)} d v(z) \\
\leq & \frac{1}{n !} \sum_{k=1}^{\infty} k^{n} e^{-(k-1)} f^{*}(w) \\
= & : C f^{*}(w) .
\end{aligned}
$$

Now we are ready to prove the theorem announced at the beginning of this section.

Theorem 6.2. Let $f \in L^{\infty}\left(\mathbb{C}^{n}\right)$. Then $\lim _{t \rightarrow 0} \tilde{f}^{(t)}(w)=f(w)$ for almost every $w \in \mathbb{C}^{n}$. In particular,

$$
\lim _{t \rightarrow 0}\left\|\tilde{f}^{(t)}\right\|_{\infty}=\lim _{t \rightarrow 0}\left\|T_{f}^{(t)}\right\|_{t}=\|f\|_{\infty} .
$$

Proof. Let $f \in L^{\infty}\left(\mathbb{C}^{n}\right), K \subset \mathbb{C}^{n}$ compact and $\delta, \varepsilon>0$. Choose $r$ sufficiently large such that $K \subset B(0, r)$ and

$$
\int_{\mathbb{C}^{n} \backslash B(w, r)} d \mu_{t}(z)<\frac{\varepsilon}{\|f\|_{\infty}}
$$

for all $t \in(0,1)$ and $w \in K$. To see that this is possible observe that for sufficiently large $|z|$ the weight $\frac{1}{(4 \pi t)^{n}} e^{-\frac{|z|^{2}}{4 t}}$ is decreasing as $t \rightarrow 0$. We may thus choose a radius $r_{0}$ such that (6.11) holds for some $w_{0} \in K$. Adding the diameter of $K$ to $r_{0}$ yields a sufficiently large radius $r$.

Cutting $f$ at that radius, i.e. setting $g_{0}:=f \cdot \chi_{B(0, r)} \in L^{1}\left(\mathbb{C}^{n}, d v\right)$, we get

$$
\left|\tilde{f}^{(t)}(w)-\tilde{g}_{0}{ }^{(t)}(w)\right|=\int_{\mathbb{C}^{n} \backslash B(w, r)}|f(w-z)| d \mu_{t}(z)<\varepsilon
$$

for all $w \in K$ and $t \in(0,1)$. Now choose a continuous function $g_{1}$ of compact support such that $\left\|g_{0}-g_{1}\right\|_{L^{1}}<\delta$. Then, by the inequality above and Proposition $\underline{3.2}$,

$$
\left|\tilde{f}^{(t)}(w)-f(w)\right| \leq\left|\tilde{f}^{(t)}(w)-{\tilde{g_{0}}}^{(t)}(w)\right|+\left|{\tilde{g_{0}}}^{(t)}(w)-{\tilde{g_{1}}}^{(t)}(w)\right|+\left|\tilde{g}_{1}^{(t)}(w)-g_{1}(w)\right|
$$




$$
\begin{aligned}
& +\left|g_{1}(w)-g_{0}(w)\right|+\left|g_{0}(w)-f(w)\right| \\
< & 2 \varepsilon+\left|\tilde{g}_{0}^{(t)}(w)-\tilde{g}_{1}^{(t)}(w)\right|+\left|g_{1}(w)-g_{0}(w)\right|
\end{aligned}
$$

for all $w \in K$ and sufficiently small $t$. To obtain the assertion, we need to show that

$$
\left\{w \in \mathbb{C}^{n}: \limsup _{t \rightarrow 0}\left|\tilde{f}^{(t)}(w)-f(w)\right|>4 \varepsilon\right\}
$$

is a null set for all $\varepsilon>0$.

By Lemma 6.1, we have

$$
\left|\tilde{g}_{0}^{(t)}(w)-\tilde{g}_{1}{ }^{(t)}(w)\right| \leq C\left(g_{0}-g_{1}\right)^{*}(w)
$$

and as is well-known, the Hardy-Littlewood maximal function satisfies the weak $(1,1)$ inequality, i.e. there exists a constant $C_{1}$ depending only on the dimension $n$ such that

$$
\left|\left\{w \in \mathbb{C}^{n}: g^{*}(w)>\varepsilon\right\}\right| \leq \frac{C_{1}}{\varepsilon}\|g\|_{L^{1}}
$$

for all $g \in L^{1}\left(\mathbb{C}^{n}, d v\right)$ (see [22, Theorem 1]). Applying this to $g_{0}-g_{1}$, we obtain

$$
\begin{aligned}
\left|\left\{w \in \mathbb{C}^{n}:\left|\tilde{g}_{0}^{(t)}(w)-\tilde{g}_{1}^{(t)}(w)\right|>\varepsilon\right\}\right| & \leq\left|\left\{w \in \mathbb{C}^{n}:\left(g_{0}-g_{1}\right)^{*}(w)>\frac{\varepsilon}{C}\right\}\right| \leq \frac{C C_{1}}{\varepsilon}\left\|g_{0}-g_{1}\right\|_{L^{1}} \\
& <\frac{C C_{1}}{\varepsilon} \delta .
\end{aligned}
$$

Moreover,

$$
\left|\left\{w \in K:\left|g_{1}(w)-g_{0}(w)\right|>\varepsilon\right\}\right| \leq \frac{1}{\varepsilon}\left\|g_{0}-g_{1}\right\|_{L^{1}}<\frac{\delta}{\varepsilon}
$$

by Markov's inequality. Thus

$$
\left|\left\{w \in K: \limsup _{t \rightarrow 0}\left|\tilde{f}^{(t)}(w)-f(w)\right|>4 \varepsilon\right\}\right|<\frac{C C_{1}+1}{\varepsilon} \delta .
$$

Since $\delta$ was arbitary, we get

$$
\left|\left\{w \in K: \limsup _{t \rightarrow 0}\left|\tilde{f}^{(t)}(w)-f(w)\right|>4 \varepsilon\right\}\right|=0
$$

for all $\varepsilon>0$ and all compact sets $K \subset \mathbb{C}^{n}$. Clearly, by taking a countable covering, this remains true if we replace $K$ by $\mathbb{C}^{n}$ in the above formula. Therefore $\tilde{f}^{(t)}(w)$ converges to $f(w)$ for almost every $w \in \mathbb{C}^{n}$.

To prove the second assertion choose for every $\varepsilon>0$ a bounded set $A_{\varepsilon} \subset \mathbb{C}^{n}$ with $\left|A_{\varepsilon}\right|>0$ such that $|f(w)| \geq\|f\|_{\infty}-\varepsilon$ for all $w \in A_{\varepsilon}$. By Egorov's theorem, we can additionally assume $\tilde{f}^{(t)}(w) \rightarrow f(w)$ uniformly for all $w \in A_{\varepsilon}$. It follows

$$
\|f\|_{\infty} \geq \lim _{t \rightarrow 0}\left\|\tilde{f}^{(t)}\right\|_{\infty} \geq \lim _{t \rightarrow 0}\left\|\left.\tilde{f}^{(t)}\right|_{A_{\varepsilon}}\right\|_{\infty} \geq\|f\|_{\infty}-\varepsilon
$$


for all $\varepsilon>0$. Using Remark 2.1 and the obvious inequality $\left\|T_{f}^{(t)}\right\|_{t} \leq\|f\|_{\infty}$, we conclude

$$
\lim _{t \rightarrow 0}\left\|\tilde{f}^{(t)}\right\|_{\infty}=\lim _{t \rightarrow 0}\left\|T_{f}^{(t)}\right\|_{t}=\|f\|_{\infty} .
$$

\section{EXAMPLES}

In this section we provide three explicit examples. The first two are counterexamples to the statements of Theorem 3.4 and Theorem 4.9. Clearly, these functions cannot be uniformly continuous or have vanishing mean oscillation. The third example shows that the semi-commutator of two unbounded Toeplitz operators can be bounded and that its norm can still tend to 0 as $t \rightarrow 0$.

(A): Direct calculation shows that a natural orthonormal basis for $H^{2}\left(\mathbb{C}, d \mu_{t}\right)$ consists of the functions

$$
e_{k}^{(t)}=\frac{z^{k}}{\sqrt{(4 t)^{k} k !}}, \quad \text { where } \quad k \in \mathbb{Z}_{+}=\{0,1,2,3, \ldots\} .
$$

Moreover, for $f(z)=\exp \left(i|z|^{2}\right)$ and $g(z)=\exp \left(-i|z|^{2}\right)$, we see that $T_{f}^{(t)}, T_{g}^{(t)}$ are diagonal in the orthonormal basis $\left\{e_{k}^{(t)}: k \in \mathbb{Z}_{+}\right\}$with eigenvalues

$$
s_{k}\left(\exp \left(i|z|^{2}\right)\right)=(1-4 t i)^{-(k+1)} \quad \text { and } \quad s_{k}\left(\exp \left(-i|z|^{2}\right)\right)=(1+4 t i)^{-(k+1)} .
$$

Since $f(z) g(z) \equiv 1$

$$
\left\|T_{f}^{(t)} T_{g}^{(t)}-T_{f g}^{(t)}\right\|_{t}=\sup \left\{1-\left(1+16 t^{2}\right)^{-(k+1)}: k \in \mathbb{Z}_{+}\right\}=1
$$

for all $t>0$. We also observe that $\left[T_{f}^{(t)}, T_{g}^{(t)}\right]=0=\{f, g\}$.

(B): Here is another counterexample to the quantization result in Theorem 3.4 and Theorem 4.9. Different from the example in (A) we choose a symbol which has high oscillation inside the domain (in a zero-neighbourhood). Such an effect was already observed in [5], however, the present example is even simpler. Let $n=1$ and consider the following symbol:

$$
f(z):= \begin{cases}0, & \text { if } z=0 \\ 1, & \text { if } 2^{j} \leq|z|<2^{j+1} \text { and } j \in \mathbb{Z} \text { is even } \\ -1, & \text { if } 2^{j} \leq|z|<2^{j+1} \text { and } j \in \mathbb{Z} \text { is odd. }\end{cases}
$$

We have $-f(z)=f\left(\frac{z}{2}\right)$ for all $z \in \mathbb{C}$. Let $U_{t}: H_{t}^{2} \rightarrow H_{\frac{1}{4}}^{2}$ be the family of isometries defined in (2.4). As was observed in (2.5) the Toeplitz operator $T_{f(\cdot 2 \sqrt{t})}^{\left(\frac{1}{4}\right)}$ transforms under conjugation by $U_{t}$ as follows:

$$
T_{f}^{(t)}=U_{t}^{*} T_{f(\cdot 2 \sqrt{t})}^{\left(\frac{1}{4}\right)} U_{t}
$$


Choose a sequence $t_{\ell}:=4^{-\ell-1}$ where $\ell \in \mathbb{N}$. Then we have

$$
T_{f}^{\left(t_{\ell}\right)} T_{f}^{\left(t_{\ell}\right)}-T_{f^{2}}^{\left(t_{\ell}\right)}=U_{t_{\ell}}^{*}\left[T_{f\left(\cdot 2^{-\ell}\right)}^{\left(\frac{1}{4}\right)} T_{f\left(\cdot 2^{-\ell}\right)}^{\left(\frac{1}{4}\right)}-I\right] U_{t_{\ell}}
$$

Because of $f\left(\cdot 2^{-\ell}\right)=(-1)^{\ell} f$ we obtain:

$$
\left\|T_{f}^{\left(t_{\ell}\right)} T_{f}^{\left(t_{\ell}\right)}-T_{f^{2}}^{\left(t_{\ell}\right)}\right\|_{t_{\ell}}=\left\|T_{f}^{\left(\frac{1}{4}\right)} T_{f}^{\left(\frac{1}{4}\right)}-T_{f^{2}}^{\left(\frac{1}{4}\right)}\right\|_{\frac{1}{4}}=\left\|T_{f}^{\left(\frac{1}{4}\right)} T_{f}^{\left(\frac{1}{4}\right)}-I\right\|_{\frac{1}{4}}=(*) .
$$

One can check (e.g. using the fact that $T_{f}^{\left(\frac{1}{4}\right)}$ is a diagonal operator) that $(*)$ is non-zero. Since $(*)$ also does not depend on $\ell$ we cannot have $\lim _{t \rightarrow 0}\left\|T_{f}^{(t)} T_{f}^{(t)}-T_{f^{2}}^{(t)}\right\|_{t}=0$.

(C): We consider the annihilation and creation operators $T_{\bar{z}}^{(t)}, T_{z}^{(t)}$ on the one-particle bosonic Fock space $H^{2}\left(\mathbb{C}, d \mu_{t}\right)$ for all $t>0$. Standard calculation shows for $k \in \mathbb{Z}_{+}$:

$$
\begin{aligned}
T_{z}^{(t)} e_{k}^{(t)} & =\{(4 t)(k+1)\}^{1 / 2} e_{k+1}^{(t)}, \\
T_{\bar{z}}^{(t)} e_{k+1}^{(t)} & =\{(4 t)(k+1)\}^{1 / 2} e_{k}^{(t)} \quad \text { and } \quad T_{\bar{z}}^{(t)} e_{0}^{(t)}=0 .
\end{aligned}
$$

It follows for $k \in \mathbb{Z}_{+}$that

$$
\left[T_{\bar{z}}^{(t)}, T_{z}^{(t)}\right] e_{k}^{(t)}=4 t e_{k}^{(t)}
$$

Noting that $T_{\bar{z}}^{(t)} T_{z}^{(t)}=T_{\bar{z} z}^{(t)}$ yields

$$
\left\|T_{z}^{(t)} T_{\bar{z}}^{(t)}-T_{z \bar{z}}^{(t)}\right\|_{t}=4 t \rightarrow 0 \quad \text { as } \quad t \rightarrow 0 .
$$

Remark. Direct computational checks of Theorem 3.4 in the diagonal case bring us to (or even over) the edge of what is possible using Stirling's approximation. For an example, consider the estimation of

$$
\left\|T_{|z|}^{(t)} T_{|z|}^{(t)}-T_{|z|^{2}}^{(t)}\right\|_{t}
$$

Acknowledgement: We thank Jingbo Xia for his useful conversation and comments. In particular, he pointed out the simple proof of Corollary 2.3 which replaced our previous (and slightly weaker) estimate on the norm of the Hankel operator $H_{f}^{(t)}$.

\section{REFERENCES}

[1] W. Bauer, Berezin-Toeplitz quantization and composition formulas, J. Funct. Anal. 256 (10) (2009), 3107-3142.

[2] W. Bauer, Mean oscillation and Hankel operators on the Segal-Bargmann space, Integr. Equ. Oper. Theory 52 (2005), 1-15.

[3] W. Bauer, L.A. Coburn, Toeplitz operators with uniformly continuous symbols, Integr. Equ. Oper. Theory 83 (2015), 24-34.

[4] W. BAuer, L.A. Coburn, Heat flow, weighted Bergman spaces and real analytic Lipschitz approximation, J. reine angew. Math. 703, (2015), 225-246.

[5] W. BAuer, L.A. Coburn, Uniformly continuous functions and quantization on the Fock space, Bol. Soc. Mat. Mex. 22 (2016), 669-677. 
[6] W. Bauer, L.A. Coburn, J. Isralowitz, Heat flow, BMO, and the compactness of Toeplitz operators, J. Funct. Anal. 259 (2010), 57-78.

[7] W. BAuer, R. Hagger, N. VAsilevski, Uniform continuity and quantization on bounded symmetric domains, in preparation, available on arXiv: 1611.09085.

[8] D. Békollé, C. Berger, L.A. Coburn, K.H. Zhu, BMO in the Bergman metric on bounded symmetric domains, J. Funct. Anal. 93 (1990), 310-350.

[9] F. A. BerezIn, Quantization, Izv. Akad. Nauk SSSR Ser. Mat. 38 (1974), 1116-1175.

[10] F. A. Berezin, Quantization in complex symmetric spaces, Izv. Akad. Nauk SSSR Ser. Mat. 39 (1975), no. 2, 363-402.

[11] F. A. Berezin, General concept of quantization, Comm. Math. Phys. 40 (1975), 153-174.

[12] M. Bordemann, E. Meinrenken, M. Schlichenmaier, Toeplitz quantization of Kähler manifolds and $\mathfrak{g l}(n), n \rightarrow \infty$ limits, Comm. Math. Phys. 165 (1994), 281-296.

[13] D. BorThwick, Microlocal techniques for semiclassical problems in geometric quantization, In: Perspectives on quantization Contemporary Mathematics, 214 (1998) 23-37. AMS, Providence.

[14] D. Borthwick, A. Lesniewski, H. Upmeier, Non-perturbative deformation quantization on Cartan domains, J. Funct. Anal. 113 (1993), 153-176.

[15] L.A. Coburn, Deformation estimates for Berezin-Toeplitz quantization, Comm. Math. Phys. 149 (1992), 415-424.

[16] M. Engliš, Weighted Bergman kernels and quantization, Comm. Math. Phys. 227 (2002), 211-241.

[17] S. KlimeK, A. Lesniewski Quantum Riemann surfaces I. The unit disc Comm. Math. Phys. 146 (1992), 103-122.

[18] M. RIEffel, Deformation quantization of Heisenberg manifolds, Comm. Math. Phys. 122 (1989), 531-562.

[19] M. Rieffel, Deformation quantization and operator algebras, Proc. Symp. Pure Math., Vol. 51, Amer. Math. Soc., Providence (1990), 411-423.

[20] D. Sarason, Functions of vanishing mean oscillation, Trans. Amer. Math. Soc. 207 (1975), 391-405.

[21] X. Shi, A. Torchinsky, Functions of vanishing mean oscillation, Math. Nachr. 133 (1987), 289-296.

[22] E.M. Stein, J.O. Strömberg, Behavior of maximal functions in $\mathbb{R}^{n}$ for large $n$, Ark. Mat., Vol. 21, No. 1-2 (1983), 259-269.

\section{WOLFRAM BAUER}

INSTITUT FÜR ANALYSIS

Welfengarten 1, 30167 Hannover, Germany

E-mail address: bauer@math.uni-hannover.de

Lewis A. Coburn

DEPARTMENT OF MATHEMATiCS

SUNY AT Buffalo, New York 14260, USA

E-mail address: lcoburn@buffalo.edu

RAFFAel Hagger

INSTITUT FÜR ANALYSIS

Welfengarten 1, 30167 Hannover, Germany

E-mail address: raffael.hagger@math.uni-hannover.de 